\title{
Peertechz
}

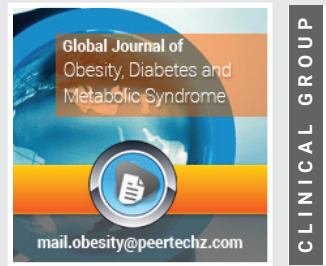

Research Article

\section{Lipid profile, abnormality of serum glucose levels and their associated factors in multidrug- resistant tuberculosis patients}

\author{
Endalkchew Biranu ${ }^{1,3}$, Mistire Wolde ${ }^{1 *}$, Abebe Edao \\ Negesso ${ }^{1}$, Million Molla Sisay ${ }^{3}$ and Habteyse Hailu Tola ${ }^{2}$ \\ ${ }^{1}$ Addis Ababa University College Of Health Sciences Department Of Medical Laboratory Sciences, \\ Addis Ababa, Ethiopia \\ ${ }^{2}$ Ethiopian Public Health Institute, Tuberculosis/HIV Research Directorate, Addis Ababa, Ethiopia \\ ${ }^{3}$ St. Peter's Specialized Hospital, Research And Evidence Generation Directorate, Addis Ababa, \\ Ethiopia
}

Received: 04 October, 2021

Accepted: 18 October, 2021

Published: 19 October, 2021

*Corresponding author: Mistire Wolde, BSc (MLT), MSc, PhD), Associate Professor, Department of Medical Laboratory Sciences, College of Health Sciences Addis Ababa University, P.O. Box 30538, Addis Ababa, Ethiopia, Tel: +251-911-69 97-10, 911699710; E-mail:mistire08@gmail.com ORCID: https://orcid.org/0000-0003-3523-7609 Keywords: Multidrug-resistant tuberculosis; Diabetes; Prediabetes; Lipid abnormalities; Lipid profile Copyright: (C) 2021 Biranu E, et al. This is an openaccess article distributed under the terms of the Creative Commons Attribution License, which permits unrestricted use, distribution, and reproduction in any medium, provided the original author and source are credited.

https://www.peertechzpublications.com

\section{Check for updates}

\section{Abstract \\ Background: The rise of multidrug-resistant tuberculosis (MDR-TB) has become a major public health concern around the world. Most patients with pulmonary tuberculosis have low body nutrients, hypocholesterolemia, and lower values were strongly associated with mortality in these patients. Representative data on lipid profile, fasting blood glucose abnormalities and their associated factors among MDR TB patients are lacking.}

Objective: To determine lipid profile, fasting blood glucose abnormalities and their associated factors among multi-drug resistant TB patients during therapy with anti-MDR-TB regimen in St.peter's Specialized Hospital Addis Ababa, Ethiopia from January to November 2020.

Methods and materials: A cross-sectional study was conducted among multi-drug resistant TB patients during MDR therapy regimen in St. Peter's Specialized Hospital in Addis Ababa, Ethiopia from January to November 2020.Using the Consecutive sampling method a total of 162 blood samples was collected in SST tubes and analyzed by using the Cobas chemistry analyzer. Data were analyzed using SPSS software version 23 packages. Simple descriptive statistics were used to present the socio-demographic and clinical characteristics of the study participants. Other associations were performed with Pearson's correlation coefficient. A p-value of < 0.05 at the $95 \%$ confidence level were considered as statistically significant in all the analyses.

Results: Mean age of the study participant was $35.9 \pm 13.6$ years. The prevalence of diabetes was $16 \%$. Out of those who had diabetes ( $9.8 \%$ ) was newly diagnosed and $6.2 \%$ already knew their diabetes status. The most common serum lipid profile abnormalities were $92.6 \% \mathrm{HDL}-\mathrm{C}$ and $35.8 \%$ had TG levels above the borderline. Among MDR-TB patients, $25.9 \%$ had a fasting blood glucose level that was higher than normal (hyperglycemic).

Conclusion: Lipid profile abnormalities and an increased risk of diabetes have been observed in patients with multidrug-resistant tuberculosis. Factors associated with abnormal blood glucose needed attention to avoid further complications and also the finding of this research calls for the implementation of active case finding of DM in patients diagnosed for MDR-TB. 


\section{Abbreviations}

BMI: Body Mass Index; BW: Body Weight; CDC: Centre for Disease Control; DM: Diabetes Mellitus; IL: Interleukin; HDLC: High Density Lipoprotein Cholesterol; HIV: Human Immunodeficiency Virus; LDL: Low Density Lipoprotein Cholesterol; MDRTB: Multidrug Resistant Tuberculosis; TC: Total Cholesterol; TG: Triglyceride; WHO: World Health Organization

\section{Introduction}

Tuberculosis (TB) is a bacterial infection caused by the bacteria Mycobacterium tuberculosis, which most usually affects the lungs. Anything that reduces the person's immunity causes the bacteria to become active [1]. Tuberculosis bacteria have intrinsic defenses against specific medications, and genetic alterations can lead to drug resistance $[2,3]$. MDR-TB is a type of tuberculosis caused by bacteria that are resistant to at least two of the most powerful first-line anti-TB treatments (drugs), isoniazid and rifampicin [4].

The emergence of multidrug-resistant tuberculosis (MDRTB) has become a global public health concern. In 2019, an estimated 10.0 million people worldwide became ill with tuberculosis. MDR/RR TB is anticipated to be discovered in 3.3 percent of new TB patients and 18 percent of previously treated cases in 2019. In total, 465000 (range: 4000 000535 000) incident cases of rifampicin-resistant tuberculosis were expected, with 78 percent having multidrug-resistant tuberculosis. Ethiopia ranks tenth among nations with a high MDR-TB burden, according to a WHO report [5].

The release of interferon, Tumor Necrosis Factor (TNF-), and other cytotoxic chemicals, as well as cell immunity and macrophage phagocytosis of Mycobacterium tuberculosis, are all part of the TB disease process. Reactive Oxygen Species (ROS) and free radicals are produced by the phagocytic activity of macrophages, neutrophils, and monocytes [6]. Advanced tuberculosis patients have significant blood levels of these free radicals and a heightened awareness of lipid peroxidation. Peroxidation is thought to be the cause of lower serum lipid awareness and tissue inflammation [7,8]. Lipids are important nutrients that influence dietary reputation as well as immunological function.If lipid profile abnormality occurred as Adeverse Drug Reaction (ADR) in TB patients could interact with conditions such as atherosclerosis, Coronary Heart Diseases (CHD) and increase morbidity and mortality among TB patients $[9,10]$.

Diabetes Mellitus (DM) is one of the most common comorbdity among tuberculosis patients. In Ethiopia, a recent study discovered a high prevalence of diabetes mellitus among tuberculosis patients [11,12]. Patients taking a second-line anti-TB medicine must have their progress checked in the lab. However, there is a paucity of information about metabolic profile abnomalties in MDR-TB patients. As a result, the current study's findings were predicted to yield empirical data on lipid profiles, fasting blood glucose abnormalities, and their associated factors among multi-drug resistant patients.

\section{Materials and methods}

\section{Study area}

This research took place in St. Peter's specialized hospital in Addis Ababa, Ethiopia, from January to November 2020. It was founded in 1953.It is managed by Ethiopia's Federal Ministry of Health (FMoH). In April 2009, it became Ethiopia's first national hospital to offer MDR-TB treatment. It served as a training center and a center of excellence. Patients come from all across the country to get treated at this hospital.During the study, 197 MDR-TB patients were treated in the hospital. Thirty of them were hospitalized [13,14].

\section{Study design and period}

A cross-sectional study was conducted in St. Peter's Specialized Hospital in Addis Ababa,Ethiopia from January to November 2020.

\section{Source population}

All MDR TB patients in St. Peter's Specialized Hospital were the source of population.

\section{Study population}

The study population included MDR- TB patients who were admitted and treated with Second line anti TB treatments at St. Peter's TB specialized hospital.

Inclusion criteria: The inclusion criteria for this study include the following:

Age between 18 - 65 years, Patients with Drug Sensitivity Testing (DST) confirmed MDR -TB, Enrolled patients with sufficient pre-treatment data that were required for this study from the medical records, and Patients volunteer to participate in the study.

Exclusion criteria: The exclusion criteria for this study include the following:

Critically sick patients (defined as those patients who need management in an Intensive Care Unit (ICU), Patients who were not initiated anti MDR tuberculosis drugs.

\section{Dependent}

Serum level of Total Cholesterol (TC), Triglyceride (TG), High Density Lipoprotein (HDL) cholesterol, Low density lipoprotein (LDL) cholesterol, and Fasting Blood Glucose (FBS) levels.

\section{Independent}

Sex, Age, Socio-economic status, Body Mass Index (BMI), Duration of anti- MDR TB drug, precence of comorbidity, types of co-morbidity.

\section{Sample size calculation}

The sample size for each specified target was computed using the Epi info sample size calculator based on a single 
population proportion. The largest sample size was estimated using hypothyroidism prevalence $(17.2 \%)$, as reported in Ethiopia a previous observational cohort study [14] .With a 95 percent confidence level and 0.06 required precision, the sample size was determined to be 151 . The overall sample size was 166 after a $10 \%$ contingency sample was taken into account. However, when four individuals refused to participate in the study, 162 patients were included.

\section{Sampling method}

Consecutive sampling methods were used until the required sample sizes were attained in the study period time.

\section{Data collection procedure}

All experts involved in data or sample collection received training on the study's goal, how to choose study participants, data confidentiality, safety, and procedures to take when collecting, transporting, processing and analizing blood samples. Then, by allocating phlebotomy, a pre-designed questionnaire was used to collect socio-demographic and other associated data. The appointed Research directorate and main investigator oversaw the data gathering process.

\section{Sample collection}

After an overnight fast, $5 \mathrm{ml}$ of peripheral venous blood was taken from each subject's cubital vein using aseptic technique in a basic gel vacationer container (9-12 hours). Within one hour of blood collection, specimens were centrifuged at 3000 rpm to separate the serum, and total cholesterol, triglyceride, HDL-cholesterol, LDL-cholesterol , and the glucose level were measured. To collect, manage, and transport the samples to the lab, the Clinical and Laboratory Standards Institute/ NCCLS (National Clinical Chemistry Laboratory Standards) recommendations were used $[15,16]$.

\section{Lipid profiles testing}

The CobasC311 analyzers were used to quantify TC, TG, HDL-C, and LDL-C. It is a computerized and fully automated system for analyzing a wide spectrum of bodily fluids.TC was analyzed by cholesterol oxidase phenol 4-aminoantipyrine peroxidase, HDL-C by direct enzymatic method, LDL-C by direct determination, VLDL-C by calculation, TG by glycerine phosphate peroxidase. FBS by Hexokinase catalyzes the phosphorylation of glucose to glucose-6-phosphate by ATP [17].

\section{Data quality assurance}

Pre-analytical stage: Includes subject preparation, sample collection, transportation and instrument maintenance. These factors should follow the recommended quality procedure to come out with quality result. For this study the specimen were collected and transported based on the recommended procedure and the instrument was stablished based on manufacturer recommended.

Analytical stage: The accuracy and precision of the approach were used to establish its diagnostic value, therefore the data generated must be reliable. As a result, commercial quality control materials were used in the same way as patient specimen testing in this investigation.

Post-analytical stage: The metabolic findings were being analyzed to see whether there was anything out of the ordinary. The results were recorded, suitably managed, and stored in a secure location.

The procedure of data collecting and laboratory testing was overseen by the primary investigator. The acquired data was verified for errors on a regular basis, and relevant actions were taken if mistakes were discovered. To ensure data quality, the data was duplicate input into separate databases (SPSS version 23) by different people.

\section{Data analysis and interpretation}

The SPSS version 23 program was used to examine the data. The baseline measurements and metabolic abnormalties of the research subjects were represented as mean SD and percentages. Tables and figures were used to convey descriptive statistics. Bivariate and multivariate logistic regression models were constructed to see if there was a significant link between the dependent and independent variables. It was decided to employ a statistically significant $\mathrm{P}$ value of less than 0.05 .

\section{Operational definitions}

MDR-TB: Refer to TB patient resistance to first line drug at least INH and Rifampicin.

Lipid Profile: Is a panel of blood tests that serves as an initial broad medical screening tool for abnormalities in lipids, such as total cholesterol, HDL-c, LDL-c and triglycerides. The normal range of total cholesterol is less than $200 \mathrm{mg} / \mathrm{dL}, \mathrm{HDL}-\mathrm{c}$ refers as 'good cholesterol'. Normal range 40-60mg/dL. LDL-c termed as 'bad cholesterol' and is considered as major risks for cardiovascular diseases its normal range $<100-130 \mathrm{mg} / \mathrm{dL}$ and Triglyceride normal range is $100-150 \mathrm{mg} / \mathrm{dL}[18,19]$.

Normal: FBG levels below $100 \mathrm{mg} / \mathrm{dl}$, without a history of diabetic medication.

Prediabetes: Refers to a level of blood glucose between 100 and $125 \mathrm{mg} / \mathrm{dl}$ with no diabetic medication.

Diabetes: Manifests when the FBG level equals or greater than $126 \mathrm{mg} / \mathrm{dl}$ [20].

\section{Result}

\section{Participants' background characteristics}

A total of 162 MDR-TB patients study participants were included in this study. Of the total MDR-TB patients, 99 $(61.1 \%)$ were males and $63(38.9 \%)$ were females. The average age was $35.9 \pm 13.6$ years (range 18 to 79 ). Co-morbidities in patients under study were $40(24.7 \%)$. From this $26(16 \%), 10$ $(6.2 \%)$ and $4(2.5 \%)$ were detected HIV, diabetes mellitus and other disease patients respectively. 16 (9.9\%) of patients had a smoking history, and 21 (13\%) had an alcohol drinking history.. 
Patients with pulmonary TB accounted for 151 (93.2\%), extrapulmonary TB for $8(4.9 \%)$, and both for $3(1.9 \%)$. The patients had a history of MDR-TB treatment in $23(14.2 \%)$ of the cases.57 (35.2\%) were married. $76(46.9 \%)$ patients were unemployed and $139(85.8 \%)$ patients resided in urban areas. $54(33.3 \%)$ of the patients their monthly income was less than 1000. Illiteracy was $22(13.5 \%)$.

Duration of anti-MDR-TB drug used were 122 (75.3\%) for more than and above three months and $40(24.7 \%)$ for less than three months (Table 1).

\section{Types of second line drugs taken by the study partici- pants}

Second line drugs taken by the study participants were 36 (22.2\%) Prothionamide (Pto) 1(0.6\%), Paraaminosalicylatesodium (PAS), 6 (3.7\%) Capreomycin $(\mathrm{Cm})$, 151(93.2\%) Levofloxacin (Lfx), 24 (14.8\%) Moxifloxacillin (Mfx), 154 (95.1\%) Cycloserine (Cs), 156 (96.3\%) Clofazimine (Cfz), 153 (94.4\%) Linezoide (Lzd), 43 (26.5\%) Delamaid (Dlm), 154 (95.1) Bedaquiline (Bdq).The drug is given based on the weight per $\mathrm{Kg}$ of the patients.

\section{Lipid profile}

Descriptive mean $( \pm$ SD), proportion: The mean level of Total cholesterol (TC) was $134 \mathrm{mg} / \mathrm{dL}( \pm 34.6)$, Triglycerides (TG) was $138.6 \mathrm{mg} / \mathrm{dL}( \pm 64.2)$, High density lipoprotein cholesterol was $37.9 \mathrm{mg} / \mathrm{dL}( \pm 13.4)$ and Low density lipoprotein cholesterol was $69.8 \mathrm{mg} / \mathrm{dL}( \pm 29.4)$, Very low density lipoprotein cholesterol was $27.6 \mathrm{mg} / \mathrm{dL}( \pm 12.9)$, Fasting blood glucose was $102.2 \mathrm{mg} /$ $\mathrm{dL}( \pm 46.7)$. Body mass index was $19.26 \mathrm{~kg} / \mathrm{m}^{2}( \pm 3.21$.), Height was1.7m( \pm 8.2$)$, and weight was $53.4 \mathrm{~kg}( \pm 10.3)$ (Table 2 ).

Lipid profile distribution by sex and age group: Figure 1 below Showed that lipid profile Distribution by sex group. There is no significant difference between male and female on lipid profile among MDR-TB patients at St.peter's hospital.

Figure 2 below Showed that lipid profile Distribution by age group. There is no significant difference between age $<36$ and $\geq 36$ on lipid profile among MDR-TB patients at St.peter's hospital.

Lipids profile (proportion): Table 3 shows the distribution of lipid profile among the study participant based on the reference value. About ( $4.3 \%)$ of the patients had border-line TC level, but there was no high TC level in the current study (Table 3).Similarly, 35(21.6\%) of the patients had border-line TG concentration, while $23(14.2 \%)$ of the patients had high TG (Table 3). Of the total patients included into this study 44 (27.2\%) had border-line HDL level, while 106 (65.4\%) were at high risk HDL. Majority $141(87.0 \%)$ of the patients had normal LDL and only $7(4.3 \%)$ had border line LDL. Moreover, $140(86.4 \%)$ of the patients had border line and $22(13.6 \%)$ had high very low density lipoprotein (VLDL). Forty two (25.9\%) patients had high level of fasting blood sugar (FBS) (Table 3).

\section{Factors associated with total cholesterol}

Age above 35 years was significantly associated with abnormal TC $(p=0.02)$. However, age was not included in
Table 1: Sociodemographic characteristics of MDR-TB patients at St.peter's specialized hospital in Addis Ababa, Ethiopia, 2020 ( $n=162$ ).

\begin{tabular}{|c|c|c|c|}
\hline Variable & & Number & $\%$ \\
\hline \multirow{2}{*}{ Sex } & Male & 99 & 61.1 \\
\hline & Female & 63 & 38.9 \\
\hline \multirow{4}{*}{ Age category } & $18-25$ & 43 & 26.5 \\
\hline & $26-35$ & 50 & 30.9 \\
\hline & $36-45$ & 37 & 22.8 \\
\hline & $\geq 45$ & 32 & 19.8 \\
\hline \multirow{2}{*}{ Residence } & Urban & 139 & 85.8 \\
\hline & Rural & 23 & 14.2 \\
\hline \multirow{2}{*}{ Marital status } & Married & 57 & 35.2 \\
\hline & Single & 105 & 64.8 \\
\hline \multirow{3}{*}{ Occupation status } & Employed & 69 & 42.6 \\
\hline & Laborer & 17 & 10.5 \\
\hline & Unemployed & 76 & 46.9 \\
\hline \multirow{4}{*}{ Education status } & Illiterate & 22 & 13.5 \\
\hline & Primary school & 45 & 27.6 \\
\hline & Secondary school & 59 & 36.2 \\
\hline & Certificate and above & 36 & 22.1 \\
\hline \multirow{3}{*}{ Income (ETB) } & $<1000$ & 54 & 33.3 \\
\hline & $1001-2000$ & 47 & 16.7 \\
\hline & $>2000$ & 81 & 50.0 \\
\hline \multirow{2}{*}{ Pervious TB history } & No & 23 & 14.2 \\
\hline & Yes & 139 & 85.8 \\
\hline \multirow{2}{*}{ House hold currently treated for TB } & YES & 12 & 7.4 \\
\hline & NO & 150 & 92.6 \\
\hline \multirow{2}{*}{ Co-morbidity } & Yes & 40 & 24.7 \\
\hline & No & 122 & 75.3 \\
\hline \multirow{4}{*}{ Types of other disease } & NO & 122 & 75.3 \\
\hline & DM & 10 & 6.2 \\
\hline & HIV & 26 & 16.0 \\
\hline & Other & 4 & 2.5 \\
\hline \multirow{2}{*}{ Non MDR-TB drugs } & Yes & 38 & 23.5 \\
\hline & No & 124 & 76.5 \\
\hline \multirow{2}{*}{ Smoking history } & Yes & 16 & 9.9 \\
\hline & No & 146 & 90.1 \\
\hline \multirow[t]{2}{*}{ Taking alcohol history } & Yes & 21 & 13.0 \\
\hline & No & 141 & 87.0 \\
\hline \multirow{2}{*}{ Duration of anti-MDR TB DRUG } & $<3$ month & 40 & 24.7 \\
\hline & $\geq 3$ month & 122 & 75.3 \\
\hline \multirow{3}{*}{ Types of TB } & EPTB & 8 & 4.9 \\
\hline & PTB & 151 & 93.2 \\
\hline & ВОTH & 3 & 1.9 \\
\hline
\end{tabular}

ETB= Ethiopian Birr, DM= Diabetes Mellitus, HIV= Human Immune Deficiency Virus, EPTB = Extra Pulmonary Tuberculosis, PTB = Pulmonarytuberculosis, MDR-TB= Multidrugresistance Tuberculosis

Table 2: Mean distribution of lipid profiles of MDR-TB patients at St.peter's specialized hospital in Addis Ababa, Ethiopia, $2020(n=162)$.

\begin{tabular}{|c|c|c|c|c|}
\hline & Minimum & Maximum & Mean (士SD) & Median (IQR) \\
\hline Total cholesterol(mg/dl) & 51.10 & 278.00 & $134.0(34.6)$ & $132.9(42.1)$ \\
\hline $\begin{array}{c}\text { Triglycerides(mg/dl) } \\
\text { High density lipoprotein } \\
\text { cholesterol(mg/dl) }\end{array}$ & 45.40 & 372.10 & $138.6(64.2)$ & $123.4(80.3)$ \\
\hline $\begin{array}{c}\text { Low density lipoprotein } \\
\text { cholesterol(mg/dl) }\end{array}$ & 9.7 & 193.00 & $69.8(29.4)$ & $65.4(37.4)$ \\
\hline $\begin{array}{c}\text { Very low density lipoprotein } \\
\text { cholesterol(mg/dl) }\end{array}$ & 9.1 & 74.40 & $27.6(12.9)$ & $24.7(16.1)$ \\
\hline \begin{tabular}{c} 
Fasting blood glucose(mg/dl) \\
\hline Height $(\mathrm{m})$
\end{tabular} & 38.4 & 502.4 & $102.2(46.7)$ & $94.8(3.8)$ \\
\hline Weight(kg) & 1.4 & 1.8 & $1.7(8.2)$ & $1.7(0.1)$ \\
\hline BMI(kg/m $\left.{ }^{2}\right)$ & 29 & 79 & $53.4(10.3)$ & $51(12)$ \\
\hline
\end{tabular}

$\mathbf{S D}=$ Standard Deviation, $\mathbf{I Q R}=$ Inter Quartile Range, $\mathbf{m g} / \mathbf{d} \mathbf{l}=$ Milligram Per Deciliters, $\boldsymbol{m}=$ meter, $\mathbf{B M I}=$ Body Mass Index, $\boldsymbol{m}=$ meter, $\mathbf{k g}=$ Kilogram, $\mathbf{k g} / \mathbf{m}^{2}=$ Kilogram Per Meter Square

Citation: Biranu E, Wolde M, Negesso AE, Sisay MM, Tola HH (2021) Lipid profile, abnormality of serum glucose levels and their associated factors in multidrugresistant tuberculosis patients. Glob J Obes Diabetes Metab Syndr 8(3): 018-028. DOI: https://dx.doi.org/10.17352/2455-8583.000053 


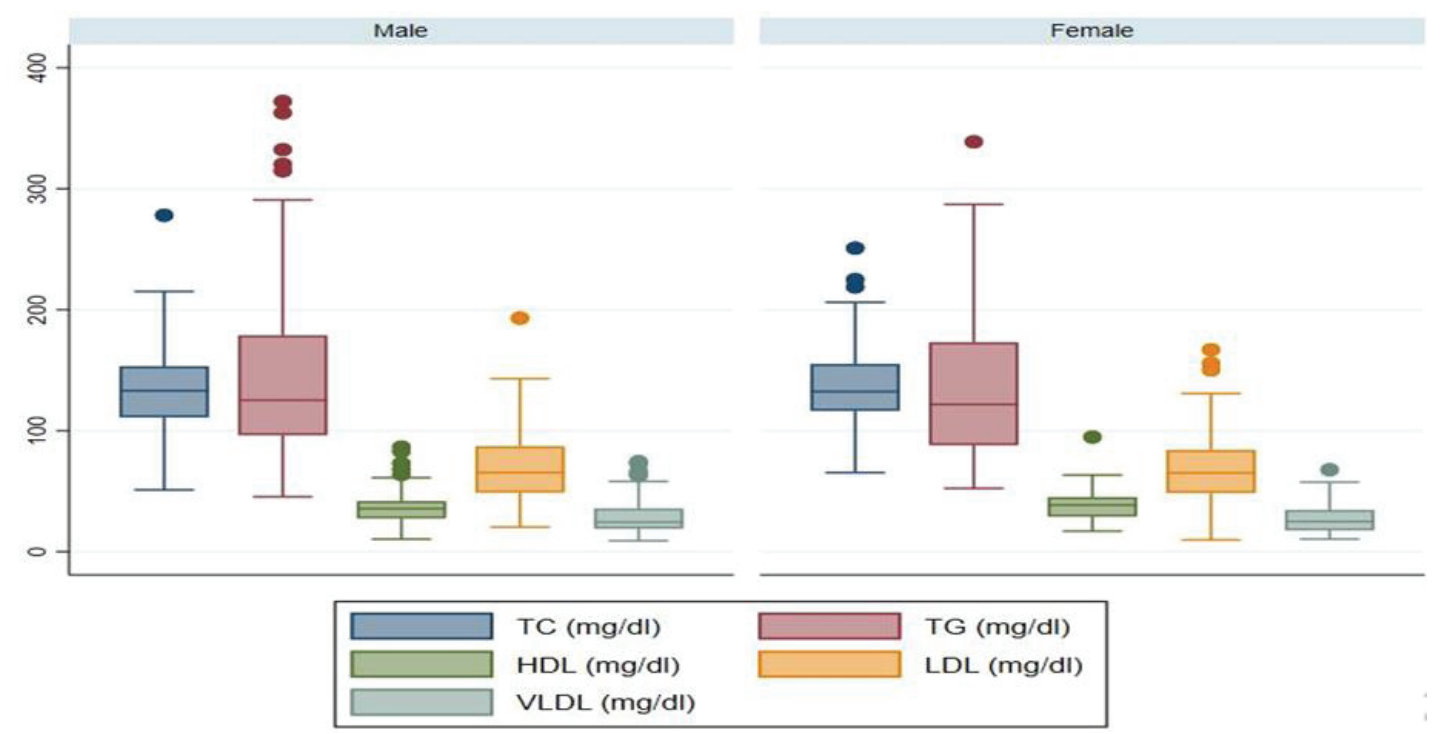

Figure 1: The box plot showing the mean distribution of lipid profile level according to sex group among MDR-TB patients at St.peter's specialized hospital.

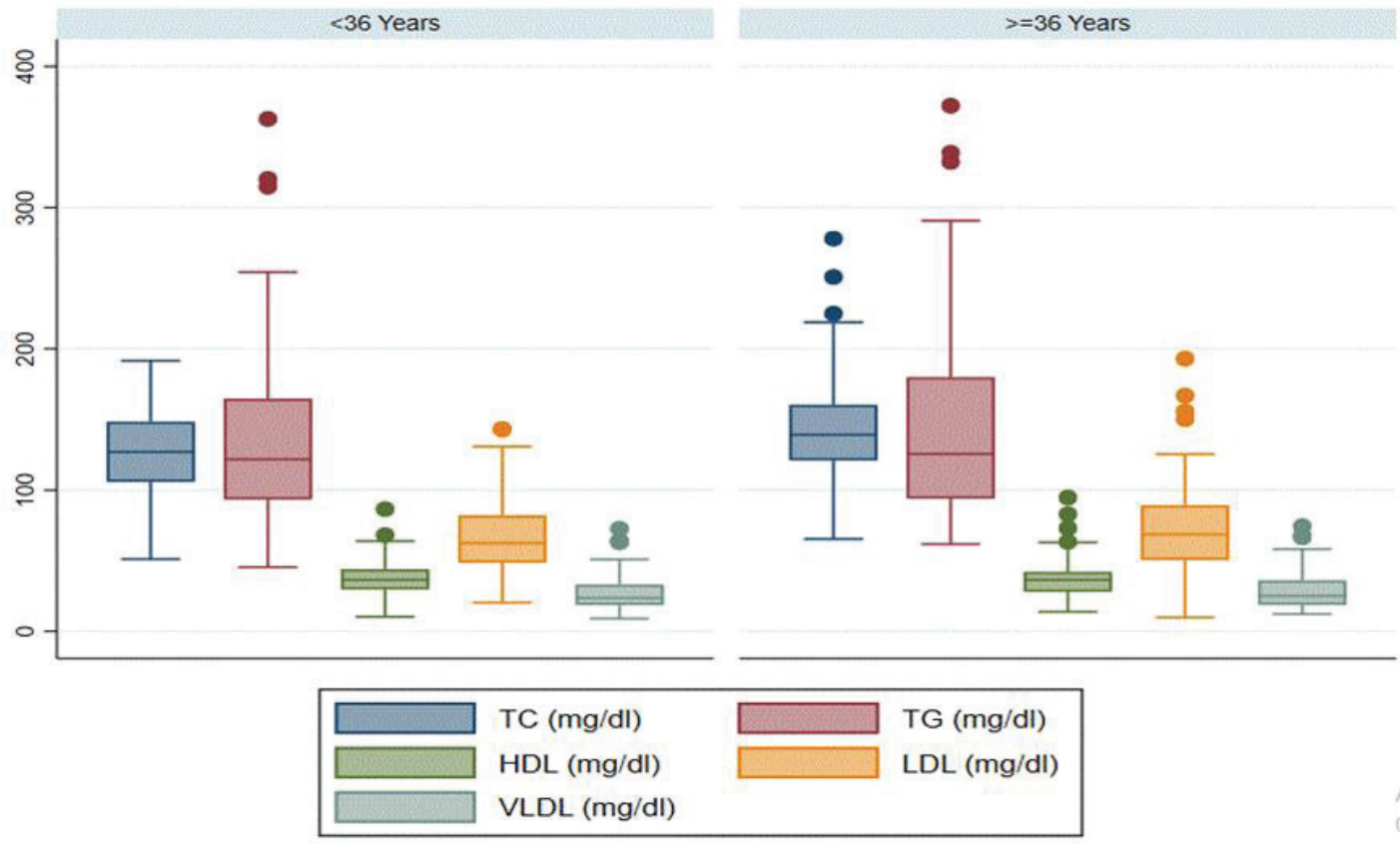

Figure 2: The box plots showing the mean distribution of lipid profile level according to age group between age $<36$ and $\geq 36$ among MDR-TB patients at St.peter's specialized hospital.

the multivariate logistic regression model because the number of abnormalities was very small (Table 4).

\section{Factors associated with triglyceride}

From the variables were assessed for the association with TG, none of them was not significantly associated with TG abnormality at the p-value less than 0.005 (Table 5).

\section{Fasting blood glucose level Distribution by sex and age group}

Figure 3 below Showed that fasting blood glucose level Distribution by sex group. There is a significant difference between male and female on fasting blood glucose level $(\mathrm{p}=0.0016)$ among MDR-TB patients at St.peter's specialized hospital.

Figure 4 below Showed that fasting blood glucose level Distribution by age group. There is no significant difference between age group $<36$ and $\geq 36$ group on fasting blood glucose level ( $\mathrm{p}=0.2281)$ among MDR-TB patients at St.peter's specialized hospital.

\section{Prevalence of diabetes and pre-diabetes among study participants}

Among the 162 MDR-TB patients who participated in this 
study $26(16 \%)$ had diabetes mellitus and $40(24.7 \%)$ were prediabetes (Figure 5). The mean value of two consecutive fasting blood glucose level of diabetes was $165.12 \mathrm{mg} / \mathrm{dl}$.

\section{Factors associated with fasting blood glucose}

Female sex $(p=0.004)$, Non MDR-TB drugs (Anti HIV drug) ( $p=0.012)$, Presence of other comorbidity $(p=0.055)$, and hypothyroidism $(p=0.002)$ were significantly associated with high fasting blood glucose level. However, these variables were not included in the final multiple logistic regression model since, the number of the abnormalities of FBS in each category were small (Table 6).

\section{Discussion}

The objective of this study was to determine the lipid profile ,fasting blood glucose level and their associated factors . The percentage distribution of the lipid parameters into normal, low, borderline and high level category was carried out among MDR-TB patient's in St.peter's specialized hospital Addis Ababa Ethiopia. The majority of the participants had a normal lipid profile and normal FBS levels, according to the findings.

In the current investigation, HIV sero-reactive patients were found in $16 \%$ of patients. These findings were lower than those reported by Meressa D, et al. 2009 (21.7 \%) in Ethiopia, who reported HIV Sero-reactive patients [14]. The study done by Kirenga J, et al. 2015 in Uganda observed HIV (41.9\%) higher than this study finding (16\%) [21]. The study done by Fisher H, et al. 2010 in USA among MDR-TB patients had lower HIV co-infection (1.54\%) observed compare with this study finding $(16 \%)[22]$.

The most frequently occurring serum lipid profile abnormalities among MDR-TB patients were HDL-C 92.6\% had above borderline value, $35.8 \%$ had above borderline TG value and $25.9 \%$ of MDR-TB patients had above the normal value (Hyperglycemic)

Table 3: Distributions of lipid abnormality among MDR -TB patients at St.peter's specialized hospital in Addis Ababa, Ethiopia, 2020 ( $n=162$ ).

\begin{tabular}{|c|c|c|}
\hline \multicolumn{2}{|c|}{ Variable } & $\mathbf{N}(\%)$ \\
\hline \multirow{2}{*}{$\mathrm{TC}$} & Normal & 155(95.7) \\
\hline & Border-line & $7(4.3 \%)$ \\
\hline \multirow{3}{*}{ TG } & Normal & 104(64.2\%) \\
\hline & Border-line & $35(21.6 \%)$ \\
\hline & High & $23(14.2 \%)$ \\
\hline \multirow{3}{*}{ HDL-C } & Normal & $12(7.4 \%)$ \\
\hline & Border-line & $44(27.2 \%)$ \\
\hline & High risk & $106(65.4 \%)$ \\
\hline \multirow{3}{*}{ LDL-C } & Normal & $141(87.0 \%)$ \\
\hline & Good & $14(8.6 \%)$ \\
\hline & Border-line & $7(4.3 \%)$ \\
\hline \multirow{2}{*}{ VLDL-C } & Border-line & $140(86.4 \%)$ \\
\hline & High & $22(13.6 \%)$ \\
\hline \multirow{3}{*}{ FBG } & Normal & $96(59.3 \%)$ \\
\hline & Hypoglycemic(L) & $24(14.8 \%)$ \\
\hline & Hyperglycemic $(\mathrm{H})$ & $42(25.9 \%)$ \\
\hline \multicolumn{3}{|c|}{$\begin{array}{l}\mathbf{N}=\text { Number, \%= Percent, } \mathbf{T C}=\text { Total Cholesterol, } \mathbf{T G}=\text { Triglyceride, } \mathbf{H D L}-\mathbf{C}=\text { High } \\
\text { Density Lipoprotein, } \mathbf{L D} \mathbf{L}-\mathbf{C}=\text { Low Density Lipoprotein Cholesterol, } \mathbf{V L D L}-\mathbf{C}=\text { Very Low } \\
\text { Density Lipoprotein Cholesterol FBG= Fasting Blood Sugar }\end{array}$} \\
\hline
\end{tabular}

Table 4: Factors associated with total cholesterol during anti-MDR -TB treatments at St.Peter's specialized hospital in Addis Ababa, Ethiopia, $2020(\mathrm{n}=162)$

\begin{tabular}{|c|c|c|c|c|}
\hline \multirow[b]{2}{*}{ Variables } & \multicolumn{3}{|c|}{ Total cholesterol } & \multirow[b]{2}{*}{ P-value } \\
\hline & & $\begin{array}{c}\text { Normal N } \\
(\%)\end{array}$ & $\begin{array}{c}\text { Abnormal N } \\
(\%)\end{array}$ & \\
\hline \multirow{2}{*}{ Sex } & Female & $96(97.0)$ & $3(3.0)$ & \multirow{2}{*}{0.432} \\
\hline & Male & $59(93.7)$ & $4(6.3)$ & \\
\hline \multirow{2}{*}{ Age (in year) } & $<36$ & $93(100)$ & $0(0)$ & \multirow[b]{2}{*}{$0.02 *$} \\
\hline & $\geq 36$ & $62(89.9)$ & $7(10.1)$ & \\
\hline \multirow{2}{*}{ Marital status } & Married & $54(94.7)$ & $3(5.3)$ & \multirow{2}{*}{0.698} \\
\hline & Single & $101(96.2)$ & $4(3.8)$ & \\
\hline \multirow{2}{*}{ Residence } & Urban & $133(95.7)$ & $6(4.3)$ & \multirow{2}{*}{1.00} \\
\hline & Rural & $22(97.5)$ & $1(4.3)$ & \\
\hline \multirow{6}{*}{ Occupation } & $\begin{array}{l}\text { Government } \\
\text { employ }\end{array}$ & $26(89.7)$ & $3(10.3)$ & \multirow{6}{*}{0.113} \\
\hline & Laborer & $17(100)$ & $0(0)$ & \\
\hline & Merchant & $39(97.5)$ & $1(2.5)$ & \\
\hline & Other & $13(81.3)$ & $3(8.8)$ & \\
\hline & Student & $22(100)$ & $0(0)$ & \\
\hline & No work & $38(100)$ & $0(0)$ & \\
\hline \multirow{4}{*}{ Education level } & Illiterate & $20(90.9)$ & $2(9.1)$ & \multirow[b]{4}{*}{0.284} \\
\hline & Primary school & 42(93.3) & $3(6.7)$ & \\
\hline & Secondary school & $57(96.6)$ & $2(3.4)$ & \\
\hline & $\begin{array}{l}\text { Certificate and } \\
\text { above }\end{array}$ & $36(1000$ & $0(0)$ & \\
\hline \multirow{3}{*}{ Monthly income } & $<1000$ & $53(96.7)$ & $1(3.3)$ & \multirow{3}{*}{0.694} \\
\hline & $1001-2000$ & $25(92.6)$ & $2(7.4)$ & \\
\hline & $\geq 2000$ & 77(95.1) & $4(4.9)$ & \\
\hline \multirow{3}{*}{ Types of MDR-TB } & EPTB & $8(100)$ & $0(0)$ & \multirow{3}{*}{1.00} \\
\hline & PTB & 144(95.4) & $7(4.6)$ & \\
\hline & Both & $3(100)$ & $0(0)$ & \\
\hline \multirow{2}{*}{ Comorbidity } & Yes & $39(97.5)$ & $1(2.5)$ & \multirow{2}{*}{1.00} \\
\hline & No & 116(95.1) & $6(4.9)$ & \\
\hline \multirow{4}{*}{ Types of disease } & HIV & $26(100)$ & $0(0)$ & \multirow{4}{*}{0.424} \\
\hline & DM & $9(90.0)$ & $1(10.0)$ & \\
\hline & OTHER & $4(100)$ & $0(0)$ & \\
\hline & NO & 116(95.1) & $6(4.9)$ & \\
\hline \multirow{2}{*}{ TSH } & Hypothyrodism & $32(100)$ & $0(0)$ & 0347 \\
\hline & NO hypothyrodism & $123(94.6)$ & $7(5.4)$ & \\
\hline Anti HIV drua & Yes & $36(94.7)$ & $2(5.3)$ & 0667 \\
\hline AntI HIV drug & No & $119(96.0)$ & $5(4.0)$ & $0.66 /$ \\
\hline Smoking history & Yes & $14(87.5)$ & $2(12.5)$ & 0143 \\
\hline & No & $141(96.6)$ & $5(3.4)$ & 0.143 \\
\hline Aloobolhictor & Yes & $21(100)$ & $2(12.5)$ & 0506 \\
\hline Alcohol history & No & $134(96.6)$ & $5(3.4)$ & 0.596 \\
\hline Duration of anti MDR- & $\geq 3$ months & 118(96.7) & $4(3.3)$ & 0.365 \\
\hline TB drugs & $<3$ months & $37(92.5)$ & $3(7.5)$ & 0.347 \\
\hline & Normal & $75(97.4)$ & $2(2.6)$ & \\
\hline BMI & Underweight & $70(94.6)$ & $4(5.4)$ & 0284 \\
\hline & Over weight & 10(90.9) & $1(9.1)$ & 0.284 \\
\hline
\end{tabular}

DM= Diabetes mellitus, $\mathbf{H I V}=$ Human Immune Deficiency Virus, EPTB= Extra Pulmonary Tuberculosis, $\mathbf{P T B}=$ Pulmonary Tuberculosis, $\mathbf{M D R}-\mathbf{T B}=$ Multidrug Resistance Tuberculosis, $\mathbf{T S H}=$ Thyroid Stimulating Hormone $\mathbf{k g} / \mathbf{m} \mathbf{2}=$ Kilogram Per Meter Square BMI= Body Mass Index * significant $p<0.005$

The study showed that the mean \pm SD $134( \pm 34.6 \mathrm{mg} / \mathrm{dL})$, $37.9( \pm 13.4 \mathrm{mg} / \mathrm{dL}), 69.8( \pm 29.4 \mathrm{mg} / \mathrm{dL})$, and $19.26 \pm 3.21 \mathrm{~kg} / \mathrm{m}^{2}$ had TC, HDL-C, LDL-C, and BMI respectively. The finding of the study higher TC $130 \pm 34.5$, Higher HDL-C $34.7 \pm 16.1$, lower LDL-C $79.03 \pm 27.5$ and comparable BMI $18.3 \pm 3.9$ with the study done by Prajapati N, et al. 2020 in Indian [23].

The study done by Alam N. et al. 2020 had mean \pm SD lower

Citation: Biranu E, Wolde M, Negesso AE, Sisay MM, Tola HH (2021) Lipid profile, abnormality of serum glucose levels and their associated factors in multidrug resistant tuberculosis patients. Glob J Obes Diabetes Metab Syndr 8(3): 018-028. DOI: https://dx.doi.org/10.17352/2455-8583.000053 
Table 5: Factors associated with triglyceride during anti-MDR -TB treatments at St.Peter's specialized hospital in Addis Ababa, Ethiopia, $2020(\mathrm{n}=162)$

\begin{tabular}{|c|c|c|c|c|c|}
\hline \multirow{2}{*}{ Variable } & \multicolumn{4}{|c|}{ Triglyceride } & \multirow[t]{3}{*}{ P-value } \\
\hline & & Normal & Abnormal & High & \\
\hline \multirow[t]{3}{*}{ Sex } & Male & & & & \\
\hline & & $62(62.6)$ & 19(19.2) & 18(18.2) & \multirow{2}{*}{0.16} \\
\hline & Female & $42(66.7)$ & $16(25.4)$ & $5(7.9)$ & \\
\hline \multirow{2}{*}{ Age (in year) } & $<36$ & $60(64.5)$ & $23(24.7)$ & $10(10.8)$ & \multirow{2}{*}{0.235} \\
\hline & $\geq 36$ & $44(63.8)$ & $12(17.4)$ & $13(18.8)$ & \\
\hline \multirow{2}{*}{ Residence } & Urban & $87(62.6)$ & $31(22.3)$ & 21(15.1) & \multirow{2}{*}{0.674} \\
\hline & Rural & 17(73.9) & $4(17.4)$ & $2(8.7)$ & \\
\hline \multirow{2}{*}{ Marital status } & Married & $37(64.9)$ & 11(19.3) & $9(15.8)$ & \multirow{2}{*}{0.655} \\
\hline & Single & $67(63.81)$ & $24(22.86)$ & 14(13.3) & \\
\hline \multirow{6}{*}{ Occupation } & $\begin{array}{l}\text { Government } \\
\text { employed }\end{array}$ & $16(55.2)$ & $8(27.6)$ & $5(17.2)$ & \multirow{6}{*}{0.377} \\
\hline & Laborer & $9(52.9)$ & $5(29.4)$ & $3(17.6)$ & \\
\hline & Merchant & $23(57.5)$ & $9(22.5)$ & $8(20.0)$ & \\
\hline & Other & $11(68.8)$ & $2(12.5)$ & $3(18.8)$ & \\
\hline & Student & $15(68.2)$ & $4(18.2)$ & $3(13.6)$ & \\
\hline & No work & $30(78.9)$ & $7(18.4)$ & $1(2.6)$ & \\
\hline \multirow[b]{4}{*}{ Education level } & illiterate & $16(72.7)$ & $5(22.7)$ & $1(4.5)$ & \multirow[b]{4}{*}{0.427} \\
\hline & Primary school & $26(57.8)$ & $11(24.4)$ & $8(17.8)$ & \\
\hline & Secondary school & $39(66.1)$ & $9(15.3)$ & 11(18.6) & \\
\hline & $\begin{array}{l}\text { Certificate and } \\
\text { above }\end{array}$ & $23(63.9)$ & $10(27.8)$ & $3(8.3)$ & \\
\hline \multirow{3}{*}{ Monthly income } & $<1000$ & $33(61)$ & $14(25.93)$ & $7(12.96)$ & \multirow{3}{*}{0.517} \\
\hline & $1001-2000$ & 14(51.9) & $7(25.90$ & $6(22.2)$ & \\
\hline & $\geq 2000$ & $57(70.4)$ & $14(17.3)$ & $10(42.3)$ & \\
\hline \multirow{3}{*}{ Types of MDT-TB } & EPTB & $3(37.5)$ & $3(37.5)$ & $2(25.0)$ & \multirow{3}{*}{0.374} \\
\hline & PTB & $98(64.9)$ & $32(21.2)$ & 21(13.9) & \\
\hline & Both & $3(100)$ & $0(0)$ & $0(0)$ & \\
\hline \multirow{2}{*}{ Comorbidity } & Yes & $23(57.5)$ & $13(32.5)$ & $4(10.0)$ & \multirow{2}{*}{0.148} \\
\hline & No & $81(66.4)$ & $22(18.0)$ & 19(15.6) & \\
\hline \multirow{4}{*}{ Types of disease } & HIV & $17(65.4)$ & $7(26.9)$ & $2(7.7)$ & \multirow{4}{*}{0.465} \\
\hline & DM & $4(40.0)$ & $4(40.0)$ & $2(20.0)$ & \\
\hline & OTHER & $3(75.0)$ & $1(25.0)$ & $0(0)$ & \\
\hline & NO & $80(65.6)$ & $23(18.9)$ & $19(15.6)$ & \\
\hline TSH & Hypothyrodism & $23(71.9)$ & $8(25.0)$ & 1(3.1) & \\
\hline 1SH & Nohypothyrodism & $81(62.3)$ & $27(20.8)$ & 22(16.9) & 0.145 \\
\hline AntiHIV drug & Yes & $23(60.5)$ & $10(26.3)$ & $5(12.5)$ & 073 \\
\hline Аाitा तार & No & $81(65.3)$ & $25(20.2)$ & $18(14.5)$ & 0.150 \\
\hline $\mathrm{Smol}+\mathrm{s}$ & Yes & $9(56.3)$ & $9(31.3)$ & $2(12.5)$ & \\
\hline & No & $95(65.1)$ & $30(20.5)$ & $21(14.4)$ & 0.541 \\
\hline Alcohol hictory & Yes & $13(61.9)$ & $5(23.8)$ & $3(14.3)$ & 094 \\
\hline 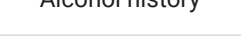 & No & $9(64.5)$ & $30(21.3)$ & $20(14.2$ & 0.94 \\
\hline Duration of anti MDR- & $\geq 3$ months & $75(61.5)$ & $29(23.8)$ & $18(14.8)$ & \\
\hline TB treatment & $<3$ months & $29(72.5)$ & $6(15.0)$ & $5(12.5)$ & 0.411 \\
\hline & Normal & $49(63.6)$ & $17(22.1)$ & $11(14.3)$ & \\
\hline $\mathrm{BMI}$ & Underweight & $49(66.2)$ & $17(23.0)$ & $8(10.8)$ & 0.312 \\
\hline & Overweight & $6(54.5)$ & $1(9.1)$ & $4(36.4)$ & \\
\hline
\end{tabular}

DM= Diabetes mellitus, HIV= Human Immune Deficiency Virus, EPTB= Extra Pulmonary Tuberculosis, $\mathbf{P T B}=$ Pulmonary Tuberculosis, $\mathbf{M D R}-\mathbf{T B}=$ Multidrug Resistance Tuberculosis, $\mathbf{T S H}=$ Thyroid Stimulating Hormone $\mathbf{k g} / \mathbf{m} \mathbf{2}=$ Kilogram Per Meter Square, BMI= Body Mass Index

TC 129.76 \pm 33.7 ; lower TG 76.09 \pm 22.8 , comparable HDL-C $35.22 \pm 13.5$, higher LDL-C 86.06 \pm 29.9 , and lower VLDL-C $15.29 \pm 4.5$ when compare to this study finding of the mean \pm SD $134( \pm 34.6 \mathrm{mg} / \mathrm{dL}), 138.6( \pm 64.2 \mathrm{mg} / \mathrm{dL}), 37.9( \pm 13.4 \mathrm{mg} /$ $\mathrm{dL})$, and $69.8( \pm 29.4 \mathrm{mg} / \mathrm{dL})$, had TC, TG, HDL-C, and LDL-C respectively [24].

The study done by Mohamed M. et al. 2012 in Egypt had mean \pm SD higher TC $148.6 \pm 30$, lower TG $80.8 \pm 23$, higher
HDL-C $53 \pm 16.5$ and higher LDL-C $79 \pm 28.6$ compare to the finding of this study which was $134( \pm 34.6 \mathrm{mg} / \mathrm{dL}), 138.6$ $( \pm 64.2 \mathrm{mg} / \mathrm{dL}), 37.9( \pm 13.4 \mathrm{mg} / \mathrm{dL})$, and $69.8( \pm 29.4 \mathrm{mg} / \mathrm{dL})$, had TC, TG, HDL-C, and LDL-C, respectively [25].

The study done by Sushilendu V, et al. 2019 in Indian the mean \pm SD of post anti TB treatment had higher TC $150 \pm 40$, lower TG $134 \pm 39$, lower $34 \pm 7$ HDL-C, and comparable LDL-C $70 \pm 14$ compare with the finding of this study which was 134 (34.6), 138.6 (64.2), 37.9 (13.4) and 69.8 (29.4) of TC, TG, HDL-C and LDL-C respectively [26].

Shvets O, et al. 2019 in Ukraine found that MDR-TB patients had lower median levels of TC, lower TG, greater HDL, and higher LDL-C compared to the current study's median level [27].

Lipids and its metabolites have beneficial effect on tuberculosis resistance through the immune system. Abnormalities in serum lipid profiles play a central role in endothelial functional abnormality which is important in the pathogenesis of atherosclerosis, insulin resistance, and hypertension. Lipoproteins rich in TG and LDL-C have been recognized to be toxic to endothelium, while HDL-C may have a protective role $[26,28]$. During inflammation, catabolism of HDL increases. In successfully treated TB patients, HDL level is supposed to increase after ATT [29].

\section{Prevalence of diabetes and pre-diabetes among MDR- TB patients}

The prevalence of pre-diabetes was ( $24.7 \%)$. This finding higher prevalence of pre-diabetes $(15.5 \%)$ and prevalence of diabetes $(16 \%)$ comparable with $(12.8 \%)$ the study done by Sarker M, et al. in 2016 in Bangladesh among TB patients [30]. The prevalence of diabetes (16\%) of current study lower when compared with (25.3\%) and comparable with the prevalence of prediabetes $(24.5 \%)$ with the study done by Viswanathan V, et al. 2012 in India among TB patients (27.5\%) [31]. Prediabetes individuals have a higher risk of developing DM; each year about $5 \%$ to $10 \%$ of prediabetes individuals develop DM [32]. This suggests that there may be an increased risk of diabetes among TB patients in the future.

\section{Risk factors of total cholesterol, triglyceride and fasting blood glucose}

The current study showed that there were not a significant association between the study characteristics and triglyceride ( $>0.05$ ). Age (in year) was found to be associated risk factors of abnormality of total cholesterol among MDR-TB patients. $(\mathrm{p}=0.02)$. The risk factors associated with fasting blood glucose were sex $(p=0.004)$, anti HIV drugs $(p=0.012)$, presence of comorbidity (0.005), types of diseases $(p=0.009)$. Certain characteristics were associated with unstable blood glucose levels. Although there were only small numbers of patients with HIV-associated TB, the diagnosis of HIV was a strong risk factor. Both HIV and TB can damage the immune system and this might result in poor glycaemia regulation [33]. Being been hypothyroidism $(\mathrm{p}=0.002)$. This finding also 

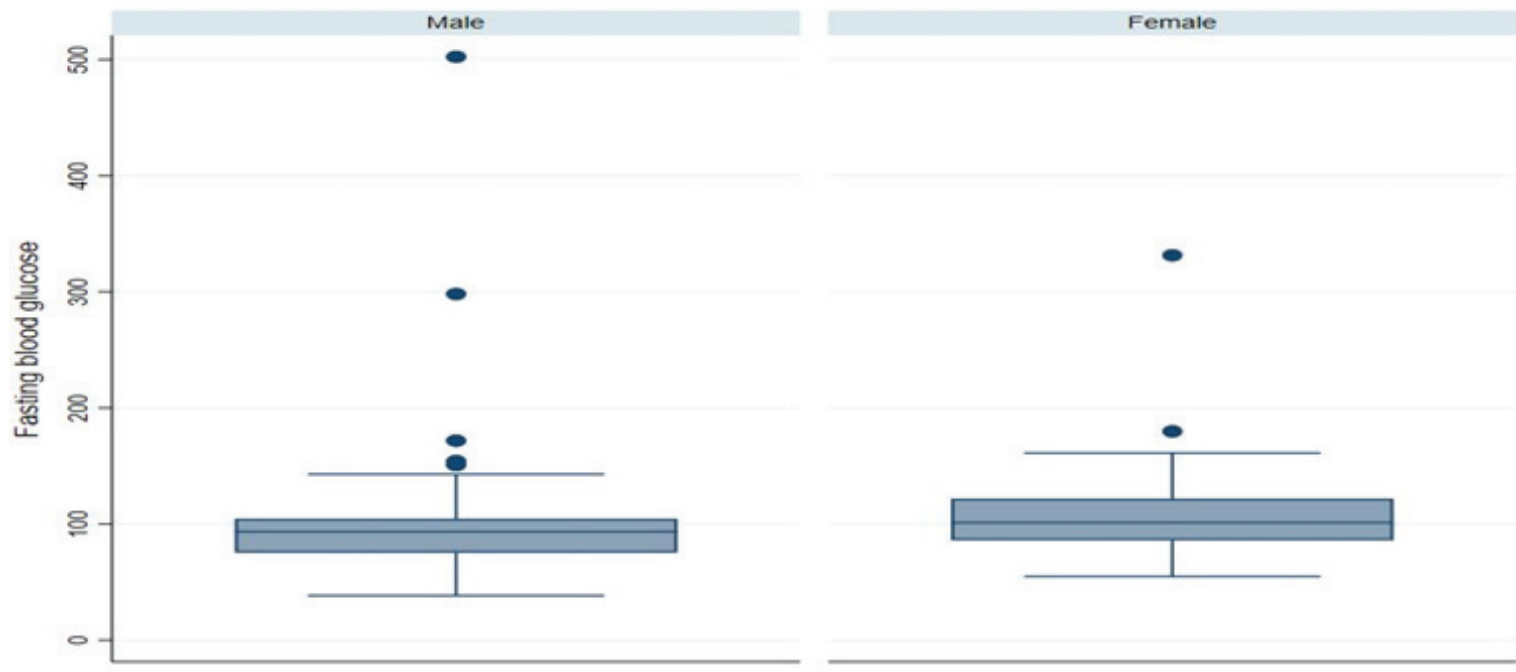

Figure 3: The box plot showing the mean distribution of fasting blood glucose level according to sex among MDR-TB patients at St.peter's specialized hospital.
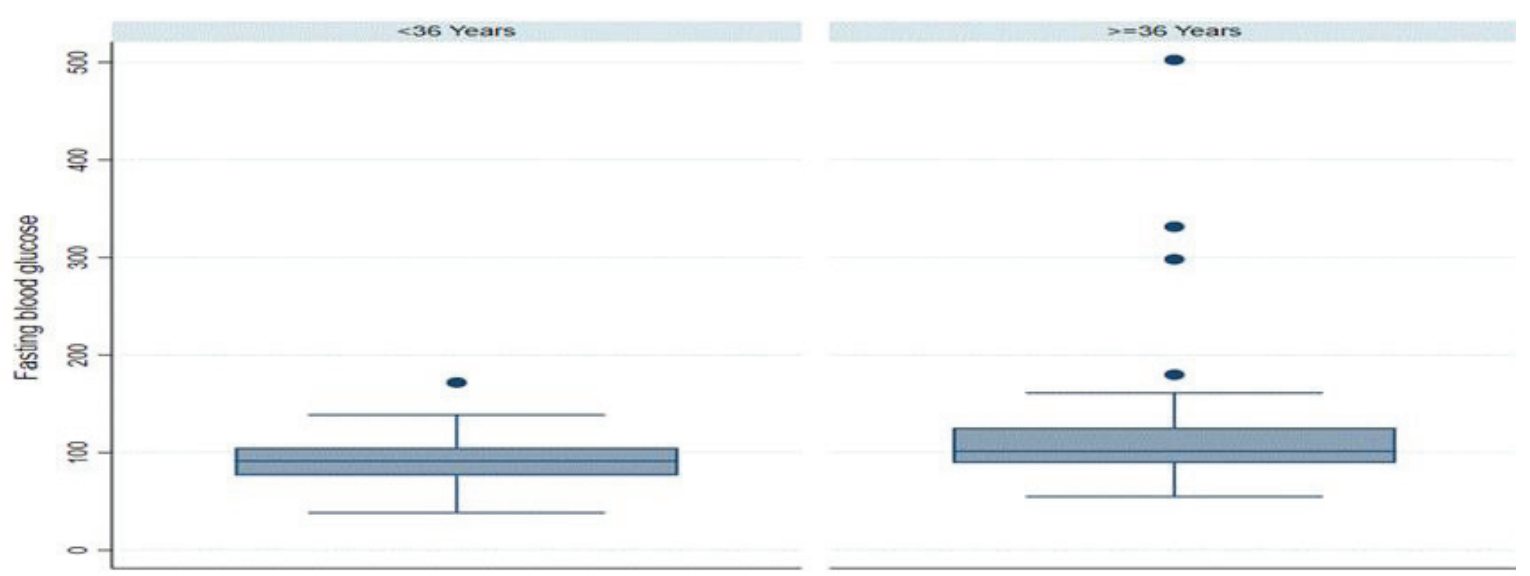

Figure 4: The box plot showing the mean distribution of fasting blood glucose level according to age group between $<36$ and $\geq 36$ among MDR-TB patients at St.peter's specialized hospital.

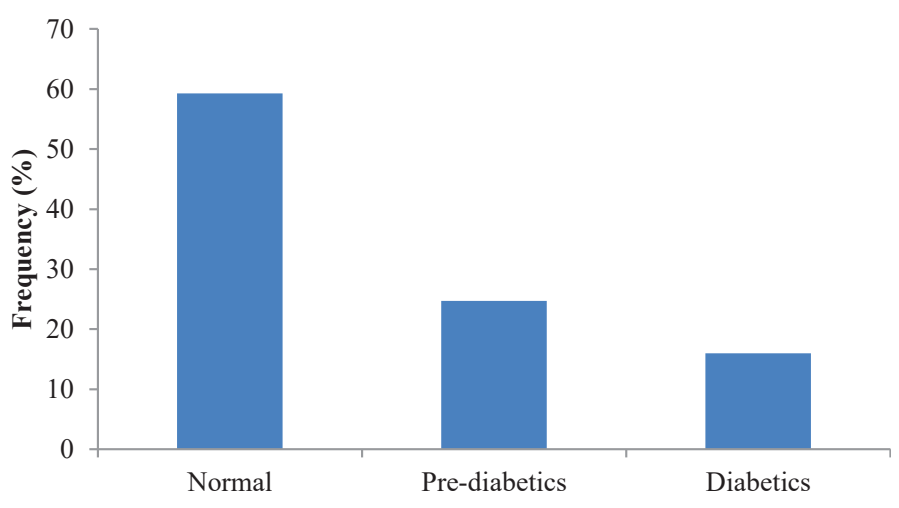

Figure 5: Distribution of prevalence of diabetes and pre-diabetes among study participants among multidrug resistance TB patients on treatment.

obsereved a research done by M. Mu noz-Torrico, et al. 2017 there was significant association hypothyroidism among DM and with out DM MDR-TB patients [34]. There are no sufficient literature about lipid profile abnormalities and associated risk factors in MDR-TB patients.

\section{Strength and limitation}

Strength: It looked at lipid profiles and fasting blood glucose levels, as well as related risk variables, in MDR-TB patients with clinical history, including categories of co-morbidity, ATT duration, and newly reported diabetes cases. The importance of combining MDR-TB risk variables of lipid profile and fasting blood glucose level abnormalities is highlighted by our findings.

Limitation: Due to economic constraints, the study was only conducted in one healthcare environment. As a result, it's tough to generalize. In addition, the study could not compare the effects of fasting blood glucose level and lipid profile variations in dietary habits, physical exercises variation.

\section{Conclusion and recommendation}

\section{Conclusion}

The study's major purpose was to look for abnormalities in lipid profiles and fasting blood glucose levels in MDR-TB patients, as well as associated risk factors. HDL-C, TG value, 
Table 6: Factors associated with fasting blood glucose during anti-MDR -TB treatments at St.Peter's specialized hospital in Addis Ababa, Ethiopia, 2020 ( $\mathrm{n}=162$ ).

\begin{tabular}{|c|c|c|c|c|c|}
\hline \multirow{2}{*}{ Variable } & \multicolumn{4}{|c|}{ Fasting blood glucose level } & \multirow{2}{*}{ p-value } \\
\hline & & Normal & Hypoglycemia & Hyperglycemia & \\
\hline \multirow{2}{*}{ Sex } & Male & $61(61.6)$ & $20(20.2)$ & $18(18.2)$ & \multirow[b]{2}{*}{$0.004^{*}$} \\
\hline & Female & $35(55.6)$ & $4(6.3)$ & $24(25.9)$ & \\
\hline \multirow{2}{*}{ Age } & $<36$ & $59(63.4)$ & $16(17.2)$ & 18(19.4) & \multirow{2}{*}{0.076} \\
\hline & $\geq 36$ & $37(53.6)$ & $8(11.6)$ & $24(34.8)$ & \\
\hline \multirow{2}{*}{ Residence } & Urban & $81(58.3)$ & $20(14.4)$ & $38(27.3)$ & \multirow{2}{*}{0.659} \\
\hline & Rural & $15(65.2)$ & $4(17.4)$ & $4(17.4)$ & \\
\hline \multirow{6}{*}{ Occupation } & Government employed & $14(48.3)$ & $3(10.3)$ & $12(41.4)$ & \multirow{7}{*}{ NA } \\
\hline & Laborer & $10(58.8)$ & $4(23.5)$ & $3(17.6)$ & \\
\hline & Merchant & $26(65.0)$ & $4(10.0)$ & $10(25.00$ & \\
\hline & Other & $5(31.3)$ & $2(12.5)$ & $9(56.3)$ & \\
\hline & Student & $16(72.7)$ & $6(27.3)$ & $0(0)$ & \\
\hline & No work & $25(65.8)$ & $24(14.8)$ & $8(21.1)$ & \\
\hline \multirow{4}{*}{ Education level } & illiterate & $10(45.4)$ & $3(13.6)$ & $9(40.9)$ & \\
\hline & Primary school & $30(66.70$ & $4(8.9)$ & $11(24.4)$ & \multirow[b]{3}{*}{0.529} \\
\hline & Secondary school & $35(59.3)$ & 11(18.6) & $13(22.0)$ & \\
\hline & Certificate and above & $21(58.3)$ & $6(16.7)$ & $9(25.0)$ & \\
\hline \multirow{3}{*}{ Monthly income } & $<1000$ & $34(62.96)$ & $12(22.2)$ & $8(14.8)$ & \multirow{3}{*}{0.119} \\
\hline & $1001-2000$ & $15(55.6)$ & $3(11.1)$ & $9(33.3)$ & \\
\hline & $\geq 2000$ & $47(58.0)$ & $9(11.1)$ & 25(30.9) & \\
\hline \multirow{2}{*}{ Marital status } & Marrieds & $32(56.1)$ & $8(14.0)$ & $17(29.8)$ & \multirow{2}{*}{0.694} \\
\hline & Single & $64(61.0)$ & $16(25.2)$ & $25(23.8)$ & \\
\hline \multirow{3}{*}{ Types of MDR-TB } & EPTB & $5(62.5)$ & $1(12.5)$ & $2(25.0)$ & \multirow{3}{*}{0.965} \\
\hline & PTB & $88(58.3)$ & $23(15.2)$ & $40(26.5)$ & \\
\hline & Both & $3(100)$ & $0(0)$ & $0(0)$ & \\
\hline \multirow{2}{*}{ Anti HIV drug } & Yes & 15(39.5) & $7(18.4)$ & $16(42.1)$ & \multirow{2}{*}{$0.012^{*}$} \\
\hline & No & $81(65.3)$ & 17(13.7) & $26(21.0)$ & \\
\hline \multirow{2}{*}{ Comorbidity } & Yes & $18(45.0)$ & $6(15.0)$ & $16(40.0)$ & \multirow{2}{*}{$0.055^{\star}$} \\
\hline & No & $78((63.9)$ & 18(15.2) & $26(21.3)$ & \\
\hline \multirow{2}{*}{ TSH } & Hypothyrodism & $11(34.4)$ & 10(31.3) & $11(34.4)$ & \multirow{2}{*}{$0.002^{*}$} \\
\hline & Nohypothyrodism & $85(65.4)$ & $14(10.8)$ & $31(23.8)$ & \\
\hline \multirow{2}{*}{ Smoking history } & Yes & $11(68.8)$ & $2(12.5)$ & $3(18.8)$ & 0011 \\
\hline & No & $85(58.2)$ & $22(15.1)$ & $39(26.7)$ & 0.811 \\
\hline 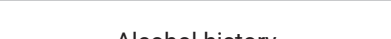 & Yes & $15(71.4)$ & $3(14.3)$ & $3(14.3)$ & 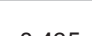 \\
\hline AICOnol nistory & No & $8(57.4)$ & $21(14.9)$ & $39(27.7)$ & 0.435 \\
\hline Duration of anti_MDR_TR treatment & $\geq 3$ months & $69(56.6)$ & 21(17.2) & $32(26.2)$ & \\
\hline 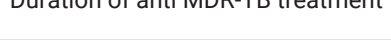 & $<3$ months & $27(67.5)$ & $3(7.5)$ & $10(25.0)$ & 0.319 \\
\hline & Normal & $52(67.5)$ & $7(9.1)$ & $18(23.4)$ & 0.150 \\
\hline BMI & Underweight & $37(50)$ & $16(21.6)$ & $21(28.4)$ & 0.152 \\
\hline & Overweight & $7(63.6)$ & $1(9.1)$ & $3(27.3)$ & \\
\hline
\end{tabular}

DM= Diabetes mellitus, HIV= Human Immune Deficiency Virus, EPTB= Extra Pulmonary Tuberculosis, $\mathbf{P T B}=$ Pulmonary Tuberculosis, $\mathbf{M D R}$-TB= Multidrug Resistance Tuberculosis, $\mathbf{T S H}=$ Thyroid Stimulating Hormone $\mathbf{k g} / \mathbf{m} \mathbf{2}=$ Kilogram Per Meter Square, $\mathbf{B M I}=$ Body Mass Index *significant $p<0.005$

and fasting blood glucose (hyperglycemia) were the most commonly found serum lipid profile abnormalities among MDR-TB patients. Diabetes and pre-diabetes are on the rise, posing a threat to TB control and necessitating a greater awareness of lifestyle changes.

\section{Recommendation}

Further research and studies are needed to determine the relationship between lipid profile abnormalities and fasting blood glucose levels with associated factors among MDRTB patients. As a result of our findings, we believe that lipid profiles and fasting blood glucose levels should be tested among MDR-TB patients for better patient management and treatment outcomes.

\section{Acknowledgment}

The authors acknowledge that the Addis Ababa University College of Health Science, the Directorate of St. Peter's Specialized Hospital Research \& Evidence Generation and the staff of St. Peter's Specialized Hospital Laboratory have given me the opportunity to complete, encourage and endorse this paper.

\section{Ethical consideration}

This study was approved by the Department of Research and Ethics Committee (DREC) of Medical Laboratory Sciences, College of Health Sciences Addis Ababa University (Approval number-DRERC/479/19/MLS) and Saint Peter specialized 
hospital (approval number-V143/28/01/2020). Informed consent was obtained from each participant, and sensitive information that could identify the patients was not disclosed to protect confidentiality, in accordance with the declaration of Helsinki.

\section{Informed consent}

The patients were told of the intent of the study and were assured of the confidentiality of the details, and the participation was entirely voluntary and no single service from the facility was missed by signing the consent form at the time of unwillingness or withdrawal.

\section{Founding}

The sources of budgets were Addis Ababa University, St.peter's hospitals and personal.

\section{Author's contributions}

All authors made a significant contribution to the work reported, whether that is in the conception, study design, execution, acquisition of data, analysis and interpretation, or in all these areas; took part in drafting, revising or critically reviewing the article; gave final approval of the version to be published; have agreed on the journal to which the article has been submitted; and agree to be accountable for all aspects of the work.

\section{References}

1. Global Tuberculosis Report (WHO) (WHO/HTM/TB/2013.11) (2013) World Health Organization, Geneva, 2013. Link: https://bit.ly/3G7LhfV

2. Louw G, Warren R, Gey van Pittius N, McEvoy C, Van Helden P, et al. (2009) Balancing Act: Efflux/Influx in Mycobacterial Drug Resistance. Antimicrob Agents Chemother 53: 3181-3189. Link: https://bit.ly/3BX70cE

3. Cooper AM (2009) Cell-mediated Immune responses in tuberculosis. Annu Rev Immunol 27: 393-422. Link: https://bit.ly/3FWasBI

4. Mishra G, Ghorpade SV, Mulani J (2014) XDR-TB: An outcome of programmatic management of TB in India. Indian J Med Ethics 11: 47-52. Link: https://bit.ly/2YY7RX0

5. WHO (2020) Global Tuberculosis Report 2020.Geneva: World Health Organization. Link: https://bit.ly/3vnbdz0

6. Sultan KM, Alobaidy W, Aljubouri AM, Naser A, Baghdad JFM, et al. (2012) Assessment of body mass index and nutritional status in pulmonary tuberculosis patients. Journal of the faculty of Medicine 54: 204-208. Link: https://bit.ly/2YUhLJg

7. Rothschild BM, Martin LD, Lev G, Bercovier H, Bar-Gal GK, et al. (2001) Mycobacterium tuberculosis complex DNA from an extinct bison dated 17,000 years before the present. Clin Infect Dis 33: 305-311. Link: https://bit.ly/3p9iaCN

8. Yamanaka K, Sakai S, Nomura F, Akashi T, Usui T (2001) A nutritional investigation of homeless patients with tuberculosis. Kekkaku (Tuberculosis) 76: 363-370. Link:https://bit.ly/3DOFJ7V

9. Yusoff FB, Haque AT, Hashim SR, Haque M, Ariffin B, et al. (2016) Lipid profile of the Coronary heart disease (CHD) patients admitted in a hospital in Malaysia. Journal of Applied Pharmaceutical Science 6: 137-142. Link: https://bit.ly/3ALYVkV
10. Cappola AR, Ladenson PW (2003) Hypothyroidism and atherosclerosis. J Clin Endocrinol Metab 88: 2438-2444. Link: https://bit.ly/3ARqn0s

11. Amare H, Gelaw A, Anagaw B, Gelaw B (2013) Smear positive pulmonary tuberculosis among diabetic patients at the Dessie referral hospital, Northeast Ethiopia. Infect Dis poverty 2: 6. Link: https://bit.ly/3jeOmRc

12. Getachew A, Mekonnen S, Alemu S, Yusuf H (2014) High magnitude of diabetes mellitus among active pulmonary tuberculosis patients in Ethiopia. Br J Med Med Res 4: 862-872. Link: https://bit.ly/3AODEa5

13. Gullele Sub Cityaaca.Addis Ababa.gov.et 2019. Link: https://bit.ly/3n4z8j3

14. Meressa D, Hurtado RM, Andrews JR, Diro E, Abato K, et al. (2015) Achieving high treatment success for multidrug-resistant TB in Africa: initiation and scale-up of MDR TB care in Ethiopia-an observational cohort study. Thorax 70: 1181-1188. Link: https://bit.ly/30s4t7s

15. Surks MI, Ortiz E, Daniels GH, Sawin CT, Col NF, et al. (2004) Scientific review and guidelines for diagnosis and management. JAMA 291: 228-238. Link: https://bit.ly/3G73rhM

16. Procedures for the collection of diagnostic blood specimens by venepuncture: Approved standard. $4^{\text {th }}$. Wayne PA: National committee for Clinical Laboratory Standards 1998. Link: https://bit.ly/3BRCZ93

17. e- Lab Doc - Roche manual. Link: https://bit.ly/2Xn3zYV

18. Lee S, Farwell AP (2016) Euthyroid sick syndrome. Compr Physiol 6: 10711080. Link: https://bit.ly/3n3tlKt

19. Expert Panel on Detection, Evaluation, and Treatment of High Blood Cholesterol in Adults (2001) Executive summary of the third report of the National Cholesterol Education Program (NCEP) expert panel on detection, evaluation, and treatment of high blood cholesterol in adults adult treatment panel III.Journal of the American Medical Association 285: 2486-2497. Link: https://bit.ly/3n2xvlY

20. American Diabetes Association (2013) Diagnosis and classification of Diabetes Mellitus. Diabetes care 36: S67-S74. Link: https://bit.ly/3FPLPXo

21. Dash M, Behera P, Kumar R (2020) Thyroid profile in pulmonary tuberculosis patients: a prospective study in a tertiary medical college of southern Odisha. International Journal of Clinical Trials 7: 66-71. Link: https://bit.ly/3paa7ph

22. Yaqoob A (2012) Subclinical hypothyroidism and its consequences. J Public Health Biol Sciences 1: 53-60.

23. Hoffman CJ, Brown TT (2007) Thyroid function abnormalities in HIV-Infected patients. Clin Infect Dis 45: 488-494. Link: https://bit.ly/3DUbHiW

24. Nawaz A, Nayak MA, Hameer ST, Kamath A, Mahale A (2019) Correlation between serum lipid fractions and radiological severity in patients with drugresistant pulmonary tuberculosis: A cross-sectional pilot study. Indian Journal of Medical Specialities 10: 99. Link: https://bit.ly/3vq7JvM

25. Mohamed M, Hesham A (2012) Lipid Profile in Tuberculous Patients: A Preliminary Report. Life Science Journal 9: 719-722. Link https://bit.ly/3pcGALr

26. Sushilendu V, Kumar N, Kumar U (2019) Study of Lipid profile in pulmonary TB cases: pre and post anti-tuberculosis treatment. Journal of Medical Science and Clinical Research 07: 211-215. Link: https://bit.ly/3AVcVIW

27. Shvets O, Shevchenko O, Nosova YaV, Hankun J, Khudaieva SA (2019) A change in lipid metabolic induces drug the second -line ant tubercular therapy. International Trends in Science and Technology 2: 33-35.

28. Wartofsky L, Dickey RA (2005) The evidence for a narrower thyrotropin reference range is compelling. $\mathrm{J}$ Clin Endocrinol Metab 90: 5483-5488. Link: https://bit.ly/30DqXCH 
29. Taparia P, Yadav D, Koolwal S, Mishra S (2015) Study of lipid profile in pulmonary tuberculosis patients and relapse cases in relation with disease severity-A pilot study. IJSAR 2. Link: https://bit.ly/3BTNAQX

30. Sarker M, Barua M, Guerra F, Saha A, Aftab A, et al. (2016) Prevalence and Factors Associated with Tuberculosis and Diabetes Comorbidity in Bangladesh. Plos One 11: 1-15. Link: https://bit.ly/3pd48zZ

31. Viswanathan V, Kumpatla S, Aravindalochanan V, Rajan R, Chinnasamy C, et al. (2012) Prevalence of Diabetes and Pre-Diabetes and Associated Risk Factors among Tuberculosis Patients in India. Plos One 7: 1-9. Link: https://bit.ly/2Xn1Vqd
32. Tabák AG, Herder C, Rathmann W, Brunner EJ, Kivimäki M (2012) Prediabetes: a high-risk state for diabetes development. Lancet 379: 2279-2290. Link: https://bit.ly/3aO5KI4

33. Harries AD, Lin Y, Satyanarayana S, Lönnroth K, Li L, et al. (2011) The looming epidemic of diabetes-associated tuberculosis: learning lessons from HIV-associated tuberculosis. Int J Tuberc Lung Dis 15: 1436-1444. Link: https://bit.ly/3aMEf1p

34. Mu noz-Torrico $\mathrm{M}$, Caminero-Luna J, Migliori GB, D’Ambrosio L, CarrilloAlduenda JL, et al. (2017) Diabetes is Associated with Severe Adverse Events in Multidrug-Resistant Tuberculosis.Arch Bronconeumol 53: 245-250. Link: https://bit.ly/3jc5tU1

\section{Discover a bigger Impact and Visibility of your article publication with}

\section{Peertechz Publications}

\section{Highlights}

* Signatory publisher of ORCID

* Signatory Publisher of DORA (San Francisco Declaration on Research Assessment)

* Articles archived in worlds' renowned service providers such as Portico, CNKI, AGRIS, TDNet, Base (Bielefeld University Library), CrossRef, Scilit, J-Gate etc.

* Journals indexed in ICMJE, SHERPA/ROMEO, Google Scholar etc.

* OAI-PMH (Open Archives Initiative Protocol for Metadata Harvesting)

* Dedicated Editorial Board for every journa

* Accurate and rapid peer-review process

* Increased citations of published articles through promotions

* Reduced timeline for article publication

Submit your articles and experience a new surge in publication services (https://www.peertechz.com/submission).

Citation: Biranu E, Wolde M, Negesso AE, Sisay MM, Tola HH (2021) Lipid profile, abnormality of serum glucose levels and their associated factors in multidrugresistant tuberculosis patients. Glob J Obes Diabetes Metab Syndr 8(3): 018-028. DOI: https://dx.doi.org/10.17352/2455-8583.000053 